EPJ Web of Conferences 40, 16002 (2013)

DOI: $10.1051 /$ epjconf/20134016002

(C) Owned by the authors, published by EDP Sciences, 2013

\title{
Hall current sensor IC with integrated Co-based alloy thin film magnetic concentrator
}

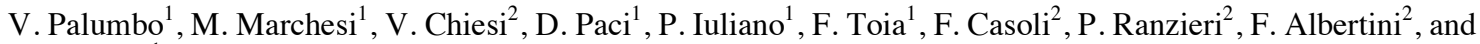 \\ M. Morelli ${ }^{1}$ \\ ${ }^{1}$ Smart Power Technology R\&D, STMicroelectronics, Via C.Olivetti 2, 20041 Agrate Brianza (MB), Italy \\ ${ }^{2}$ Institute of Materials for Electronics and Magnetism (IMEM), CNR, Parco Area delle Scienze 37/A, 43124 Parma, Italy
}

\begin{abstract}
This work deals with a cobalt-based alloy thin film magnetic concentrator (MC) which is fully integrated on a Hall sensor integrated circuit (IC) developed in the $0.35 \mu \mathrm{m}$ Bipolar CMOS DMOS (BCD) technology on 8 " silicon wafer. An amorphous magnetic film with a thickness of $1 \mu \mathrm{m}$, coercitive field $H_{c}<10 \mathrm{~A} / \mathrm{m}$ and saturation magnetization $\left(\mu_{0} M_{S}\right)$ of $0.45 \mathrm{~T}$ has been obtained with a sputtering process. The Hall sensor IC has shown sensitivity to magnetic field at room temperature of 240V/AT without concentrator and 2550V/AT with concentrator, gaining a factor of 10.5. A current sensor demonstrator has been realized showing linear response in the range -50 to $50 \mathrm{~A}$.
\end{abstract}

\section{Introduction}

In recent years an increasing interest in contactless current sensors for energy metering and hybrid or full electric vehicles and compass feature for mobile consumer applications has pushed the development of integrated Hall magnetic sensors. Two remarkable advantages of Hall current sensors with respect to the classical method of measuring a voltage drop on a shuntresistor are the higher compactness and the lower power consumption (in particular for high current systems) and the possibility to guarantee a good galvanic isolation between the system to be measured (for high voltage systems) and the control electronics.

In order to improve the sensitivity, in commercial Hall sensor ICs a thick magnetic concentrator is generally placed over the IC surface with a post-process (mechanical bonding and etching, or electroplating).

In this work, for the first time, a magnetic concentrator thin film has been integrated directly into a BCD [1] technology using standard 8" CMOS equipments, reducing the cost and improving the control of the manufacturing process.

\section{Hall sensors IC design}

\subsection{Three axis Hall sensors}

Hall sensor integrated circuits are widely used as one, two or three axes magnetic field detectors. The basic element of an Hall sensor is the Hall cell, a 4-contacts ntype diffused resistance in which a current is forced to
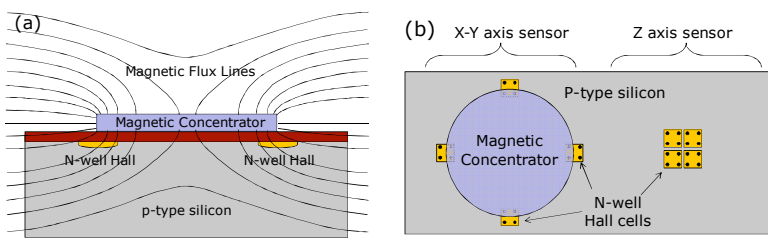

Fig. 1. (a) Cross section of an $X-Y$ axis Hall sensor. (b) Top view of a XYZ axis integrated Hall sensor realized with a $X Y$ axis sensor and $\mathrm{Z}$ axis sensor, the last one with centroid symmetric multicell layout to reduce offset and noise.

flow between two opposite contacts and a voltage described by the Lorentz's law is measured between the other two contacts when a magnetic field is applied $[2,3]$.

Such Hall cells are sensitive to the magnetic field component normal to silicon surface $B_{z}$ so in order to measure the other two components (parallel to the silicon surface) the most adopted technique is to bend the parallel components towards the normal direction by means of a high-permeability ferromagnetic concentrator [4]. In commercial devices the magnetic concentrator is generally a $10-100 \mu \mathrm{m}$ thick disc placed on the Hall sensor IC surface. Four groups of Hall cells are located at the disc edges along the $\mathrm{X}$ and $\mathrm{Y}$ directions [5].

Depending on the material permeability and on the shape factor the magnetic flux lines will converge at one disc edge and will diverge at the opposite edge. Hence, near the disc edges a normal component of magnetic field is present also if only a parallel magnetic field is applied (Fig.1a). A couple of Hall cells positioned under the disc edges will experience a magnetic field upward component at the flux convergence side and a downward

This is an Open Access article distributed under the terms of the Creative Commons Attribution License 2.0, which permits unrestricted use, distribution, and reproduction in any medium, provided the original work is properly cited. 
component at the flux divergence side, so a proper signal treatment will twice the Hall output reducing the impact of a common-mode $\mathrm{z}$-axis magnetic field. Therefore it is possible to integrate into the same chip a three-axis magnetic sensor: the XY axes by means of four Hall cells with magnetic concentrator and the $\mathrm{Z}$ axis with one group of Hall cells (Fig.1b).

\subsection{Hall cells design}

The Hall effect electric field due to the interaction of carriers with a current density $\boldsymbol{J}$ and a magnetic field $\boldsymbol{B}$ can be written as

$$
\boldsymbol{E}_{\boldsymbol{H}}=-R_{H}[\boldsymbol{J} \times \boldsymbol{B}]
$$

where $R_{H}$ is defined as the Hall coefficient [6]. The Hall voltage present between two contacts having areas $S_{I}$ and $S_{2}$ can be expressed by

$$
V_{H}=\int_{S_{1}}^{S_{2}} \boldsymbol{E}_{\boldsymbol{H}} d \boldsymbol{s}
$$

In an integrated Hall cell realized in a silicon n-type well on a p-type substrate, two opposite contacts i.e. along the $\mathrm{X}$-axis are used to inject a bias current $I_{\text {bias }}$. Between the other two contacts along y-axis a Hall voltage $V_{H}$ proportional to the magnetic field normal to silicon surface $B_{z}$ is measured. The equation (2) can be written as

$$
V_{H}=-\frac{s_{H} G}{\text { end }} B_{z} I_{\text {bias }}
$$

where $n$ and $d$ are the doping concentration and the thickness of the n-well respectively, $G$ is a geometrical correction factor and $s_{H}$ is the scattering factor of silicon usually 1.15 [7]. Reducing the thickness $d$ of the Hall cell, not only a higher $V_{H}$ is obtained but also a lower cross-talk effect respect to the other magnetic field directions. Electrons are confined in the $x-y$ plane and they can only be displaced by a $\mathrm{z}$ direction magnetic field, other directions will produce negligible effects.

An important figure of merit for a Hall cell is the sensitivity as a function of the biasing current, defined as

$$
S_{I}=\left|\frac{V_{H}}{B_{Z} I_{\text {bias }}}\right|=\frac{s_{H} G}{\text { end }}
$$

A complete evaluation of the Hall effect was performed with Cedrat Flux [8] for electromagnetic physics simulation combined with Comsol Multiphysics [9] for electrical physics simulation.

An optimum trade-off between the sensitivity and the maximum bias voltage of $3.3 \mathrm{~V}$ compatible with the CMOS logic available, has been found with four couples of $34 \times 34 \mu \mathrm{m}^{2}$ four-contacts Hall cell two by two connected in parallel to reduce offset, with a phosphorous well doping of about $10^{16}$ at $/ \mathrm{cm}^{3}$, obtaining a sensitivity of $240 \mathrm{~V} / \mathrm{AT}$.

\subsection{Magnetic Concentrator design}

The design of a fully integrated magnetic concentrator requires a large number of geometrical parameters to be defined: thickness, vertical distance from Hall cells, shape and profile. The thickness is controlled by the sputtering process, the distance from silicon is given by the thickness of dielectrics of metal interconnections, the shape is defined by the lithography process and the profile is the result of the beneath dielectrics morphology.

An apparent key parameter for the Hall sensor performances is the magnetic concentrator thickness. In fact, a concentrator with a thickness of tens of microns will guarantee a greater dynamic range with respect to a thin concentrator but no advantages in terms of sensitivity because the magnetic flux lines in both cases are bended in a similar way. The simulation results of a Hall cell with a magnetic concentrator radius disc of $125 \mu \mathrm{m}$ and a thickness of $1 \mu \mathrm{m}$ and $2 \mu \mathrm{m}$ are reported in Fig. 3. No differences are observed in the Hall voltage by applying an external magnetic field in the $\mathrm{x}$ direction (parallel to disc) up to $2 \mathrm{mT}$. On the other hand, due to the demagnetization effect the behaviour in terms of saturation magnetic field is strongly dependent on the thickness.

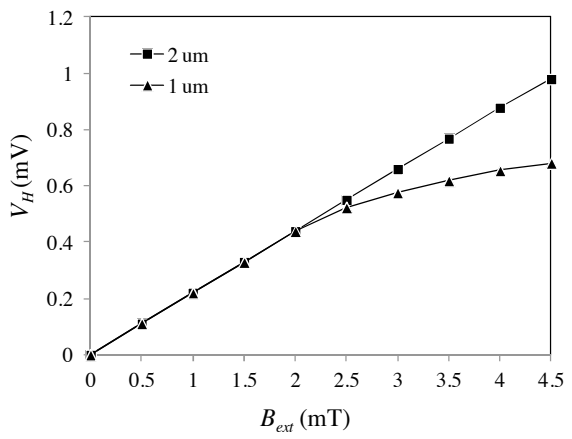

Fig. 3. Hall cell output voltage for different thickness of the magnetic concentrator disc with radius of $125 \mu \mathrm{m}$ (simulations).

This can be explained considering a thin disc as an ellipsoid where the semiaxis along the $\mathrm{z}$ direction is the film thickness $t$, and the other two semiaxes are equal to the disc radius $R$. For low thickness values, $m=t / R \ll 1$ and the disc can be considered as an oblate spheroid $[10,11]$, so the demagnetization factor in the $\mathrm{z}$ direction is larger than in $\mathrm{x}, \mathrm{y}$ directions $\left(D_{z}>>D_{x}=D_{y}\right)$. Hence, the magnetic saturation is preferentially reached in $x-y$ directions. Moreover, the magnetic field necessary to saturate in $\mathrm{x}-\mathrm{y}$ plane increases with $m$ : the higher the $m$ ratio (high thickness or little radius), the higher the demagnetization, and the saturation magnetization is reached for higher external magnetic field. For $1 \mu \mathrm{m}$ thick disc with radius of $125 \mu \mathrm{m}$ the saturation occurs for an external field of about $2 \mathrm{mT}$.

The most important advantage of an integrated process is the possibility to design the magnetic concentrator with an almost arbitrary shape with a submicron lithography capability.

Some shapes were simulated in order to maximize the sensor sensitivity. In Fig.4, simulations of the normal component of magnetic field on the single cell surface are represented for three different concentrator shapes: circular, single toothed wheel and double toothed wheel.

The double toothed wheel shape (Fig.4c) gives a not uniform distribution but a higher mean value: $28 \%$ higher than the disc shape and $6 \%$ higher than the single toothed wheel. 

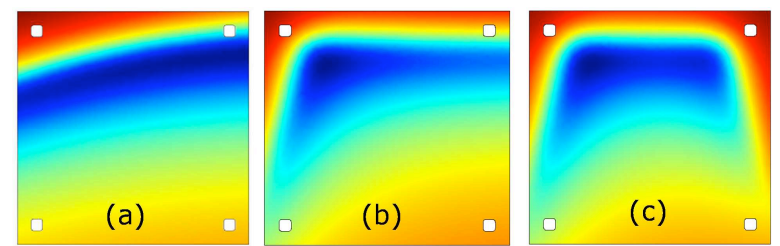

Fig. 4. Simulation of magnetic field on single Hall cell surface in case of circular (a), toothed wheel (b) and double toothed wheel (c) shape of the magnetic concentrator. The colour scale (a.u.) indicates a higher field in blue and lower in red.

\section{Experimental}

The magnetic thin film was deposited with magnetron sputtering at room temperature. The concentrator shape on the IC sensor was defined through lithographic and wet etch processes.

The magnetic material surface analysis was done through AFM (Atomic Force Microscopy). The room temperature magnetic properties and magnetic microstructure of films were investigated by alternating gradient force magnetometry AGFM and magnetic force microscopy MFM in order to observe perpendicular magnetic signals and magnetic domains. Magnetization curves of the films grown on silicon surface were measured by AGFM with magnetic field applied in the film plane.

The Hall sensor has been characterized with an experimental setup based on a couple of Helmholtz coils driven by a current generator. The magnetic field direction has been defined with the rotation of the sensor with respect to the coils axis.

\section{Results and Discussion}

\subsection{Structural-morphological characterization}

The amorphous and zero-magnetostriction Co- $16 \% \mathrm{Si}$ $9 \% \mathrm{~B}-4 \% \mathrm{Fe}-3 \% \mathrm{Mo}-1 \% \mathrm{Nb}$ (at.\%) alloy was chosen for the magnetic concentrator presented in this work. An optimization of the sputtering process was done in order to obtain an amorphous film with soft magnetic characteristics, which means low coercitivity, low saturation field, high Curie temperature, high magnetic permeability. The amorphous state is very important in order to have high magnetic permeability and high linearity of the sensor.

Film structure was investigated by TEM (Transmission Electron Microscopy) analysis (not showed here) which demonstrated that as-grown films were amorphous with a $2 \mathrm{~nm}$ coherence zone. The obtained material had a very flat surface with a roughness lower than 2nm (Fig.5a), which is compatible with amorphous structure. At high sputtering power the presence of grains aggregates was observed on a very flat surface as typical of high energy deposition process.

\subsection{Magnetic characterization}

In soft magnetic materials the presence of stress induced during the film growth can give rise to a contribution to magnetic anisotropy out of the film plane causing the enhancement of coercitivity and of saturation field.

The presence of stress can be avoided through the optimization of the deposition parameters and the thermal treatments at temperatures lower than the crystallization one $[12,13]$. The fingerprint of stress presence is the transcritical shape in the hysteresis loops [14,15] as shown in Fig.6a and a magnetization organized in weak stripe domains $[14,16]$ as reported in Fig.5b.

After a thermal treatment at $400^{\circ} \mathrm{C}$ the stress is released and the magnetization lies in the film plane. The corresponding hysteresis loop does not show the transcritical loop shape (Fig.6b) nor a MFM signal.

The saturation magnetization obtained through $\mathrm{AGFM}$ is $\mu_{0} M_{S}=0.45 \mathrm{~T}$ the coercivity is lower than 10 $\mathrm{A} / \mathrm{m}$, and saturation field is around $600 \mathrm{~A} / \mathrm{m}$. These are optimum values for the required application.

At all the sensor process steps the magnetic characteristics were measured in order to avoid the crystallization of material or the induction of stress.
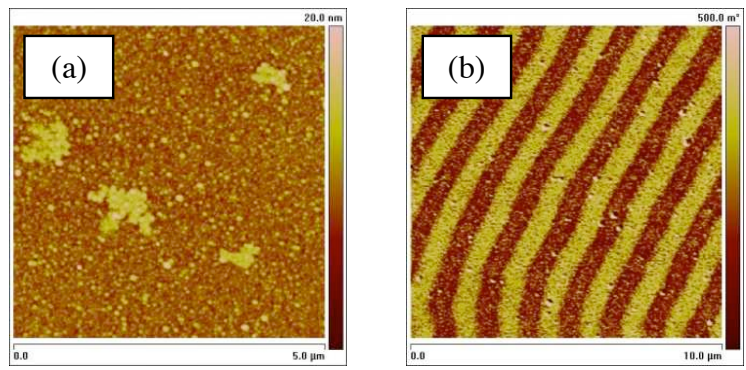

Fig. 5. (a) AFM surface image, (b) MFM domain pattern of the same sample on a larger zone.
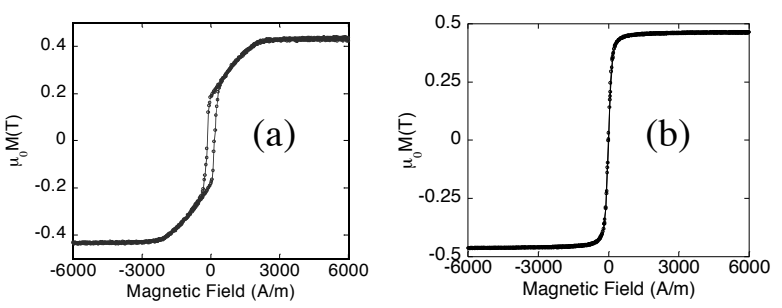

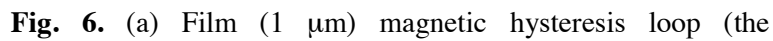
transcritical shape is due to stress induced during the film growth). (b) Film hysteresis loop after heat treatment at $400^{\circ} \mathrm{C}$.

\subsection{Hall Sensor IC Electrical characterization}

The results of the Hall sensor IC (Fig.7a) with four couples of $34 \times 34 \mu^{2}$ cells, connected in parallel to reduce the offset, with a double toothed wheel shape magnetic concentrator disc with a thickness of $1 \mu \mathrm{m}$ and radius of 125 and $250 \mu \mathrm{m}$ are reported in Table 1 . For each direction of magnetic field ( $\mathrm{X}$ and $\mathrm{Y}$ ) the output voltage of the two opposite group of Hall cells were subtracted in order to eliminate common-mode along the $\mathrm{Z}$ axis and to reduce noise and offset effects, then amplified 100x.

In Fig.8a the results of output voltage for the Hall sensor with MC (radius $250 \mu \mathrm{m}$ ) with an applied magnetic field up to $1 \mathrm{mT}$ and different biasing current values are shown. 

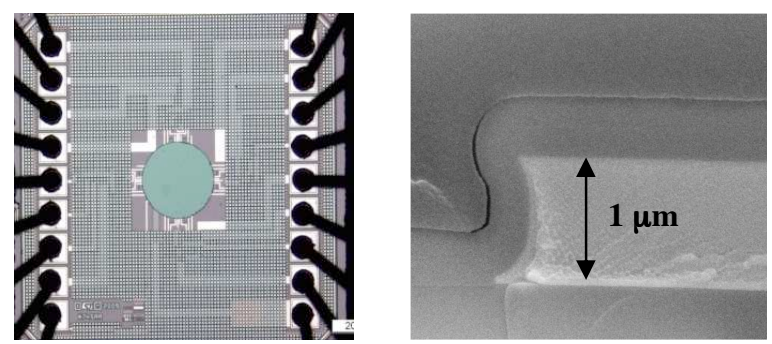

Fig. 7. (a) Photography of the Hall sensor, and (b) cross section of the magnetic concentrator.

In order to evaluate the capability of the sensor for compass applications, an accurate analysis with a little magnetic field in the earth magnetic field range $(\sim 50 \mu \mathrm{T})$ [17] has been done (Fig. 8b).

The gain of the sensor with magnetic concentrator (MC) can be evaluated as the ratio of sensitivities in $X$ and $\mathrm{Y}$ direction with the $\mathrm{MC}$ and the sensitivity of the same Hall sensor without $\mathrm{MC}$ in $\mathrm{Z}$ direction. As reported in Table 1, our Hall sensor with MC has reached a gain of 10.5 in both the directions.
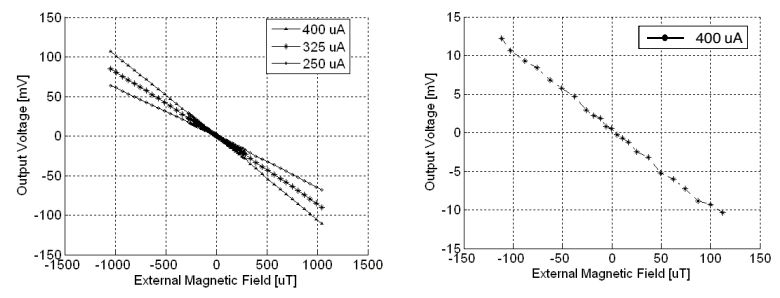

Fig. 8. Hall sensor output voltage with $\mathrm{X}$-axis magnetic field at different biasing current (a), and in the range of earth magnetic field $(\sim 50 \mu \mathrm{T})$.

\begin{tabular}{|c|c|c|}
\hline Disc Radius $(\mu \mathrm{m})$ & 125 & 250 \\
\hline X-axis Sensitivity, $S_{I}{ }^{(X)}(\mathrm{V} / \mathrm{AT})$ & $1315 \pm 25$ & $2550 \pm 45$ \\
\hline Y-axis Sensitivity, $S_{I}{ }^{(Y)}(\mathrm{V} / \mathrm{AT})$ & $1310 \pm 25$ & $2540 \pm 45$ \\
\hline X-axis Magnetic Gain, $S_{I}{ }^{(X)} / S_{I, 0}{ }^{(Z)}$ & $5.4 \pm 0.1$ & $10.5 \pm 0.1$ \\
\hline Y-axis Magnetic Gain, $S_{I}{ }^{(Y)} / S_{I, 0}{ }^{(Z)}$ & $5.4 \pm 0.1$ & $10.4 \pm 0.1$ \\
\hline Linear Error $(\% \mathrm{FS})$ & $<1.5 \%$ & $<1.5 \%$ \\
\hline Magnetic Field linear range $(\mathrm{mT})$ & \pm 2 & \pm 1 \\
\hline
\end{tabular}

Table 1. Hall sensor results at $I_{B I A S}=400 \mu \mathrm{A}$.

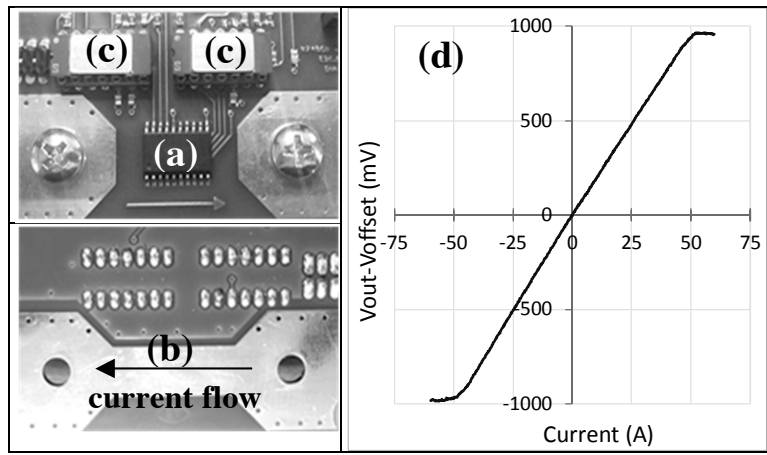

Fig. 9. Top and bottom view of PCB current sensor demonstrator with Hall sensor IC (a), the metal strip (b) and the INA (c). Output characteristics (d).
A current sensor demonstrator has been realized with a single axis Hall sensor with MC (radius $125 \mu \mathrm{m}$ ). The sensor measures the magnetic field generated by the current to be measure flowing in a metal strip on PCB with a well known geometry (Fig.9). The Hall signal was processed with a proper instrumentation amplifier (INA) with an amplification of 500 and a chopping technique. The sensor has shown a linear response with maximum output voltage of $\pm 900 \mathrm{mV}$ (with a linear error $<1.5 \%$ ) in the range of $\pm 45 \mathrm{~A}$. The saturation is reached with a magnetic field of $2 \mathrm{mT}$ at about $50 \mathrm{~A}$.

\section{Conclusions}

In this work we have demonstrated the possibility to integrate a cobalt rich amorphous soft-magnetic thin film as magnetic concentrator into a 8" silicon BCD technology Hall sensor IC. Thickness, shape and thermal treatments effects on morphological and magnetic properties of the concentrator have been investigated. The obtained sensitivity to magnetic field at room temperature was 240V/AT without concentrator and 2550V/AT with concentrator, gaining a factor of 10.5 .

\section{Acknowledgments}

A part of the work has been performed in the project ENIAC JU $\mathrm{E}^{3} \mathrm{CAR}$ co-funded by grants from: the German Federal Ministry of Education and Research (BMBF); the Belgian IWT-Flanders; the French Ministère de l'Économie, de l'Industrie et de l'Emploi - Direction Générale de la Compétitivité, de l'Industrie et des Services (DGCIS); Enterprise Ireland; the Italian Ministero Istruzione Università Ricerca - APRE Agenzia per la Promozione della Ricerca Europea; the Dutch SenterNovem; the Research Council of Norway; the Spanish DGI-Ministerio de Educación y Ciencia; the Finnish Funding Agency for Technology and Innovation (TEKES); the Czech Research and Development Council in cooperation with the Ministry of Education, Youth and Sport (R\&D); the Austrian Research Promotion Agency (FFG).

The authors would like to thank C. Riva, A.Votta, S. Pirotta, F. Milanesi, S. Antolini, F. Casati, P. Bompieri, P. Monge, I. Bianchi, P. Krejsa for their precious contribution.

\section{References}

1. D. Riccardi, $19^{\text {th }}$ ISPSD Proceedings, 73-76 (2007)

2. Z. B. Randjelovic, M. Kayal, R. Popovic, H. Blanchard, J. Solid State Circuits 37, 151 (2002)

3. M. Motz et al., IEEE Sensors 1-3, 1008-1011 (2006)

4. R. S. Popovic, P.M. Drljaca, C. Schott, R. Racz, $37^{\text {th }}$ Int. Conf. on Microelectronics (2001)

5. C.Schott, IEEE Sensors 1-2, 959 (2005)

6. R. S. Popovic, Sens. and Actuators 85, 9-17 (2000)

7. M. Paun, J. Sallese, M. Kayal, $18^{\text {th }}$ MIXDES (2011)

8. http://www.cedrat.com/en/software/flux.html

9. http://www.comsol.com/products/multiphysics/

10. D. P. Jackson, Am. J. Phys. 74, 272 (2006)

11. J. S. Ma, et al., Electr. Material Letters, 3, 87 (2007)

12. M. Coisson et al. J. Mag. Mag. Mat. 321, 806 (2009)

13. P. Sharma et al. Phys Rev. B 73, 052401 (2006)

14. R. S. A. Hubert Magnetic Domains (Springer, 2009)

15. L.M. Alvarez-Prado et al. Physica B 343, 241 (2004)

16. A. Chezan et al., J. Mag. Mag. Mat. 299, 219 (2006)

17. A. Bashirotto et al., IEEE Trans. Instr. Meas. 58, 3269 (2009) 